opalescence gradually disappears as the gel particles become smaller with increasing concentration. Gelatin jelly is therefore a gelatinous precipitate of gelatin of at least 0.7 per cent. concentration.

The size of the particles can be increased by allowing them to grow by spontaneous evaporation of the solution, subject to the necessary precautions. After one month the precipitate is buff-coloured, and appears as a mass of perfectly spherical microscopic grains exactly like Perrin's grains of mastic. From these and many similar experiments, in conjunction with the ultramicroscopic appearance, it may be concluded that a gelatin jelly is a mass of ultramicroscopic spherites of gelatin in which, as von Nägeli suggested, the water is held by molecular forces. These forces are the cause of the swelling in water, and the heat of swelling can be calculated roughly on this supposition. The structure fits exactly Zsigmondy's analysis of the vapour pressure isotherms. Experiments on the relation between the excess concentration and the size of particle are being made and may lead to a more definite form of von Weimarn's equation. But, in its present state, the formula is sufficient to explain the occurrence ${ }^{-t}$ of gelatin in the colloidal state. The molecular weight is unknown, but Dakin's recent analyses suggest that it may be as great as 10,00o, or more, a value which would correspond to a molecular diameter of $0.75 \mu \mu$ and bring its molecules up to colloidal size. But, though the molecular weight should be much less, there is no doubt that the molecules are very complex, for this is the reason for the very low diffusion constant; moreover, as the viscosity also of the sols is considerable, the factor $\mathrm{K}$ must be very large. In addition to this, the solubility, $\mathrm{L}$, is very small, and the excess concentration, $\mathrm{P}$, is usually large, so that everything conspires to produce a maximum value of $\mathrm{N}$ corresponding to the colloid condition. The permanence of the jelly is due to the very small diffusion constant, which prevents recrystallisation. But this does occur slowly, as is shown by the gradual appearance of opalescence, and even of microscopic spherites, in gels kept for a long time in sealed tubes.

Since agar-agar also separates from solution in the form of spherites, it appears that the structure of gels of this substance and of gelatin is probably that of a pile of shot, while soap and fibrinogen gels may be fibrillar. Such a fine-grained structure is compatible with all the known properties of gels, except the heat of swelling of gelatin, $5 \cdot 7$ cal. per grm., and the so-called thermal anomaly. Re-determination of the former gave $33.25 \mathrm{cal}$., and investigation of the latter showed that it was unfounded. Two questions remain undecided: (a) The nature of the spherites and $(b)$ whether they are joined together to produce a framework in the jelly. Spherites are known in every gradation, from the obviously crystalline form, built up of coarse radiating crystalline needles separately visible, through stages showing only a more or less radiating formation, but giving the well-known shadow-cross in polarised light, to apparently homogeneous spherical bodies giving no definite evidence of crystalline structure. Gelatin and agar-agar spherites appear to belong to the last class. Experimental evidence suggests that the spherites coalesce during gelation. They would seem either to aggregate crystallographically or to adhere by their mutual attraction; or, the apparent attraction may be due to the water molecules having a greater mutual attraction than the spherites. It will be admitted, however, that, in the case of such small particles, there can be very little difference between the two former methods of attachment, since the union must be due to the forces between the few molecules in the surface of contact. With two grains only, the coupling would be unstable, but it would become firm as more grains were added.

Since writing the above, evidence has been obtained that the gelatin spherites are really crystalline. Some of these, grown to a size of about $3 \mu$, by methods previously described, and mounted in glycerin, were examined with polarised light. When the Nicols were crossed they became brilliantly coloured and many showed shadow crosses, while grains of mastic prepared by Perrin's method and mounted in the same way became invisible. These experiments are being continued, but, without evidence to the contrary, it will be difficult to deny that gelation is merely an extreme case of crystallisation.

\title{
Physical Properties of Clay and Clay-Mud.
}

MUD and clay are materials, the properties of which are not only of concern to the meticulous housewife and to the children who make mud pies and clay engines; the geologist has found interest in their formation, and from the study of them is able to trace a large part of the history of the earth's crust. They have played their part in the rsthetic development of the race. They have been the architect's and engineer's friend for the making of building materials, and have filled them with concern and not infrequently dismay when they have desired to build upon them or when they desired to support them. The story of the development of buildings, bridges, and other types of structures, tells of many failures, because of the treacherousness and uncertainty of these materials, and partly, at least, because engineers and architects had not attempted to determine their properties in a scientific manner.

Mr. A. S. E. Ackermann has presented, during recent years, four papers to the Society of Engineers in which he has described experiments to determine the physical properties of clay and the effect of water content upon their properties. ${ }^{1}$ He has shown that, like certain metals, clays have a certain measure of fluidity. When a disc resting on clay is loaded

1 Society of Engineers Transactions, I919-20-2I-22. the disc sinks into the clay, the amount it descends depending on the load and on the time allowed; and when the load exceeds a certain amount, which depends upon the amount of water present, the rate and extent of penetration are considerably increased. The stress at which this occurs, Mr. Ackermann has called the pressure of fluidity. Mr. Ackermann's experiments have been directed toward determining the bearing power of soils, and the loads that can safely be applied to them.

The difficulty of reconciling experimental data on the properties of these materials is evidenced by comparing the results of experimenters. Mr. Ackermann states that the friction angle for wet mud varies as the square root of the pressure, while Crosthwaite says it is proportional to the square root of the pressure. A special committee of the American Society of Civil Engineers to codify present practice on the bearing values of soils for foundations, has issued a series of reports, and has emphasised the importance of the colloid content of clay, which consists of non-crystalline, hydrated, gelatinous aluminium silicates, gelatinous silicic and hydrated ferric oxides; rarely aluminium hydrate may also be present. Most of the grains of the minerals in the clay are enveloped by colloid, but quartz grains 
do not, as a rule, have the colloid coating. The plasticity of the clay depends upon the amount of colloid present. To separate the colloidal from the granular material, the clay is revolved at 40,000 revolutions per minute in a separator.

Dr. Hubert Chatley, in a recent paper, ${ }^{2}$ has discussed the properties of clay-mud, and states that it has three special features:

(I) A granulated structure of varying degrees of fineness.

(2) A semi-permanent water content, which gives it peculiar mechanical properties.

(3) A certain small reserve of chemical potential, which, under certain conditions, will cause it to change in various ways.

He discusses the methods of observing the granular matter by means of the microscope, and states that the plasticity depends upon the size of the products ${ }^{2}$ Society of Engineers, June 1922. and the proportion of colloids present. He divides the water content into three classes.

Clay-mud containing is per cent. by weight of water has a tensile strength of $\mathrm{I}_{5} \mathrm{lb}$. per sq. inch, but doubling the water content reduces the tensile strength to one-third of this amount. With 28 per cent. of water, its viscosity is about the same as a heavy grease, corresponding to a shear strain of $I$ radian per Ioo seconds, under a shear stress of more than Ioo grm. per sq. cm. It differs from heavy grease, however, in that water is extruded as the pressure is increased. It is not watertight, and dykes allow water to percolate very slowly, but if the surface of the dyke is dry, the surface tension may arrest the flow.

The results of the data indicate agreement with common experience that the water content of clay is of great importance, and they also indicate that, as with all other materials, the working stresses should be within the " elastic range."

\section{Silvanus Thompson Memorial Lecture.}

$A \mathrm{~T}$ the request of the Finsbury Technical College $A$ Old Students' Association, Sir Oliver Lodge gave the first of these lectures at the College on February I, Sir Charles Parsons in the chair, to an audience numbering more than a thousand and including many eminent past students. After a reference to the splendid work of the College in the past, and its hopes for the future, the lecturer recalled the brilliant succession of teachers-Ayrton, Perry, Meldola-colleagues of Thompson. Of the latter he said: "The breadth of his outlook and width of his interests are almost proverbial ; his facility in foreign languages enabled him to hold his own in assemblies abroad, and he had a real artistic faculty. He had a love of discoveries in their nascent stages, and became a recognised historian of science. To a man of his cosmopolitan feelings and pacific disposition, the war and its atrocities were a great distress; grief and worry and overwork overtook him, and he succumbed on June I2, I9I6-a victim of the warhaving been principal of Finsbury since $188_{5}$."

Proceeding to the subject of the lecture, "The Origins or Foundations of Wireless Communication," and confining himself to matters prior to I896, Sir Oliver recalled that the term "inductance" did not at first exist ; Lord Kelvin introduced it as a mathematical coefficient, Maxwell spoke of self-induction, and Heaviside originated the term now used. In the early work on the production and detection of electric waves in the ether, Kelvin, Maxwell, FitzGerald, and Hertz laid the foundations which made the present superstructure possible.

In 1875 Edison observed the possibility of drawing sparks from insulated objects in the neighbourhood of an electrical discharge; already in 1842 , Henry, in Washington, had surmised-through a similar observation - that there was some similarity between the etherial disturbance caused by the discharge of a conductor and the light emitted from an ordinary high-temperature source. Early in the 'eighties David Hughes, working with the microphone and galvanometer, got something like a coherer, but was discouraged from pursuing the matter. In I865 Maxwell gave the theory of electric waves, before their generation or detection was understood; he showed that they would travel with the velocity of light, that light was an electromagnetic phenomenon, that conductors of electricity must be opaque to light, and that the refractive index of a substance was intimately related to its dielectric coefficient.
This discovery aroused great enthusiasm, and one result was to influence the lecturer to devote his life to the study of electric waves; he discussed them with Fleming and FitzGerald, and spoke about them at the British Association in 1879 and later. In I 883 FitzGerald proposed the generation of the waves by using the oscillatory discharge of a Levden jar, and the lecturer, in 1887 , produced and detected them. The waves were received on wires adjusted to the right length for resonance. The experiments of Hertz, who received the waves on a nearly closed ring of wire having a short spark gap, were reported by FitzGerald at the British Association meeting of I888, and Sir Oliver calculated the horse-power of the oscillator-about Ioo, for a millionth of a second ; he exhibited many of the effects of the waves at the Royal Institution in I889, but there was nothing akin to signalling; that was foreshadowed, in I892, together with the possibility of tuning, by Sir William Crookes, who spoke of wave-lengths with which to signal to specific people, and alluded to Hughes's signals made from room to room without intervening wire.

In I89o Sir Oliver employed a form of coherer to complete a bell circuit, and in I 893 heard of Branly's filings-coherer. In memory of Hertz, for whom Sir Oliver expressed the greatest admiration, both on account of his experimental skill and mathematical thoroughness, he gave a lecture at the Royal Institution on the work of Hertz; at this lecture actual signalling with a coherer was demonstrated. This work led to the grant of Lodge's patent in the United States, which was the fundamental patent of the American Marconi Company. The lecture stimulated Dr. Muirhead, Captain (now Admiral Sir Henry) Jackson, Admiral Popoff, Prof. Righi, and others to their experimental successes; in I896 Mr. Marconi came to this country-and the rest is common knowledge.

After the lecture the audience was entertained at a conversazione in the laboratories. A beautiful collection of Dr. Thompson's paintings was on view, together with a number of his works, including a translation of Gilbert's "De Magnete" (I60I) and a copy of the original. Coils constructed by Faraday, the first Nicol prism, a coherer made by Sir Oliver Lodge, acoustical and optical models, and many personal relics were lent by the late Doctor's family. Mr. W. M. Mordey, president of the Old Students' Association of the College, gave a demon- 